\title{
Silica nanoparticles induce autophagy and endothelial dysfunction via the PI3K/Akt/mTOR signaling pathway
}

Junchao Duan ${ }^{1,2}$
Yongbo Yu',2
Yang Yu'
Yang Li',
Ji Wang
Weijia Geng
Lizhen Jiang
Qiuling Li',
Xianqing Zhou',2
Zhiwei Sun
'School of Public Health, Capital
Medical University, Beijing, ${ }^{1, B e i j i n g}$
Key Laboratory of Environmental
Toxicology, Capital Medical University,
Beijing, People's Republic of China

This article was published in the following Dove Press journal:

International Journal of Nanomedicine

5 November 2014

Number of times this article has been viewed

\begin{abstract}
Although nanoparticles have a great potential for biomedical applications, there is still a lack of a correlative safety evaluation on the cardiovascular system. This study is aimed to clarify the biological behavior and influence of silica nanoparticles $\left(\mathrm{Nano}_{-} \mathrm{SiO}_{2}\right)$ on endothelial cell function. The results showed that the $\mathrm{Nano}-\mathrm{SiO}_{2}$ were internalized into endothelial cells in a dose-dependent manner. Monodansylcadaverine staining, autophagic ultrastructural observation, and LC3-I/LC3-II conversion were employed to verify autophagy activation induced by $\mathrm{Nano}-\mathrm{SiO}_{2}$, and the whole autophagic process was also observed in endothelial cells. In addition, the level of nitric oxide (NO), the activities of NO synthase (NOS) and endothelial (e)NOS were significantly decreased in a dose-dependent way, while the activity of inducible (i)NOS was markedly increased. The expression of C-reactive protein, as well as the production of proinflammatory cytokines (tumor necrosis factor $\alpha$, interleukin [IL]-1 $\beta$, and IL-6) were significantly elevated. Moreover, $\mathrm{Nano}-\mathrm{SiO}_{2}$ had an inhibitory effect on the phosphoinositide 3-kinase (PI3K)/protein kinase B (Akt)/mammalian target of rapamycin (mTOR) signaling pathway. Our findings demonstrated that $\mathrm{Nano}_{-} \mathrm{SiO}_{2}$ could disturb the NO/NOS system, induce inflammatory response, activate autophagy, and eventually lead to endothelial dysfunction via the $\mathrm{PI} 3 \mathrm{~K} / \mathrm{Akt} / \mathrm{mTOR}$ pathway. This indicates that exposure to $\mathrm{Nano}-\mathrm{SiO}_{2}$ is a potential risk factor for cardiovascular diseases.
\end{abstract}

Keywords: silica nanoparticles, endothelial dysfunction, autophagy, nitric oxide, inflammation

\section{Introduction}

Numerous engineered nanomaterials, including metal nanoparticles, magnetic nanoparticles, quantum dots, and silica nanoparticles $\left(\mathrm{Nano}-\mathrm{SiO}_{2}\right)$, have promising applications in the delivery of drugs, proteins, vaccines, and nucleotides. ${ }^{1,2}$ Compared with other nanoparticles, $\mathrm{Nano}-\mathrm{SiO}_{2}$ are one of the most widely-used nanomaterials due to their favorable optical properties, high hydrophilicity, large surface area, fantastic modification, and good biocompatibility. ${ }^{3}$ Benefitting from the development of nanotechnology, the global market of nanotechnology-based industries will invest beyond US\$2.6 trillion in the year $2015 .{ }^{4}$ In contrast, the research funding that focuses on the safety evaluation of nanomaterials gets only $4 \%$ of the United States Food and Drug Administration budget. ${ }^{5}$ It should be noted that nanotoxicology is not only a government problem, but also an issue of human health. However, there is still a lack of evaluation regarding the toxicity of nanomaterials.

Currently, attention is being paid to the relationship between nanoparticles and autophagy. A growing body of literature suggests that autophagy dysfunction may 
contribute to the toxicity of nanomaterials. ${ }^{6}$ Autophagy is defined as a protective mechanism in cells that can degrade proteins and/or damaged organelles to maintain cellular homeostasis. ${ }^{7}$ Nevertheless, the process of autophagy can be stimulated by different kinds of microorganisms, such as bacteria, viruses, or parasites. Since nanoparticles are similar in size to some microorganisms, it is possible that nanoparticles can be perceived as a foreign body and activate autophagy in cells. ${ }^{8}$ Protecting nanocarriers from degradation before reaching the target organs is always a challenge in the design of efficient and safe nano-based delivery systems. ${ }^{9}$ Being delivered intravenously into the target organs or cells makes nanocarrier contact with the systemic circulation unavoidable. ${ }^{10}$ Whether nanomaterials can influence endothelial cell function is a crucial issue in human health and safety.

Endothelial dysfunction, first described by Panza et $\mathrm{al}^{11}$ and Endemann and Schiffrin ${ }^{12}$ in 1990, is defined as a series of events, mainly including vasodilation reduction, proinflammatory response, and prothrombic properties. Endothelial dysfunction particularly refers to the reduction of vasodilation that is closely related to the decrease of nitric oxide (NO) bioavailability. ${ }^{13}$ Yet, the biological behavior and toxic effects of nanoparticles on the vasculature is still poorly understood. In this study, we confirmed the association between Nano$\mathrm{SiO}_{2}$-induced autophagic activity and endothelial dysfunction by conducting a series of assessments in the primary human umbilical vein endothelial cells (HUVECs), such as cellular uptake, ultrastructural observation, monodansylcadaverine (MDC) staining, microtubule-associated protein 1 light chain $3 \beta$ (LC3) conversion, NO and NO synthase (NOS) system, and proinflammatory cytokine expression. We also examined the PI3K/Akt/mTOR signaling pathway to explore the possible mechanism of the toxicity induced by Nano- $\mathrm{SiO}_{2}$. These findings provide persuasive evidence for the toxic effects of nanomaterials on the cardiovascular system.

\section{Materials and methods \\ Preparation and characterization of $\mathrm{Nano}_{-} \mathrm{SiO}_{2}$}

Nano- $\mathrm{SiO}_{2}$ were prepared and characterized as described in our previous studies. ${ }^{14,15}$ Briefly, $2.5 \mathrm{~mL}$ of tetraethylorthosilicate was added to a premixed ethanol solution $(50 \mathrm{~mL})$ containing ammonia $(2 \mathrm{~mL})$ and water $(1 \mathrm{~mL})$. The reaction mixture was kept stirring (150 rotations $[\mathrm{r}] /$ minute) at $40^{\circ} \mathrm{C}$ for 12 hours. The particles were isolated by centrifugation (12,000 r/minute) for 15 minutes and rinsed for three times; they were then dispersed in deionized water $(50 \mathrm{~mL})$. The size and shape of Nano- $\mathrm{SiO}_{2}$ was observed under transmission electron microscopy (TEM) (JEM-2100; JEOL, Tokyo, Japan). The size distribution of Nano-SiO 2 was performed by ImageJ software (National Institutes of Health, Bethesda, MD, USA). In addition, the zeta potential and hydrodynamic sizes of Nano-SiO ${ }_{2}$ were detected with a Zetasizer (Nano ZS90; Malvern Instruments, Malvern, UK). Prior to experimental use, the Nano-SiO 2 were dispersed under a sonicator for 5 minutes (Bioruptor ${ }^{\circledR}$ UCD-200; Diagenode s.a., Liege, Belgium). The red fluorescent-labeled Nano- $\mathrm{SiO}_{2}$ was prepared and characterized in our previous study ${ }^{16}$ and used for the cellular uptake experiment only.

\section{Cell culture experiment}

The HUVECs line was purchased from the Cell Resource Center, Shanghai Institutes for Biological Sciences (Shanghai, People's Republic of China). The cells were cultured in a humidified environment $\left(37^{\circ} \mathrm{C} ; 5 \% \mathrm{CO}_{2}\right)$, maintained in Dulbecco's Modified Eagle's Medium (DMEM) (Gibco ${ }^{\circledR}$; Thermo Fisher Scientific, Waltham, MA, USA) with 10\% fetal bovine serum (Gibco ${ }^{\circledR}$; Thermo Fisher Scientific), $100 \mathrm{U} / \mathrm{mL}$ of penicillin, and $100 \mu \mathrm{g} / \mathrm{mL}$ of streptomycin. For all tests, the HUVECs were seeded in six-well plates $\left(1 \times 10^{5}\right.$ cells $\left./ \mathrm{mL}\right)$ and allowed to attach for 24 hours; they were then exposed to different concentrations $(25 \mu \mathrm{g} / \mathrm{mL}$, $50 \mu \mathrm{g} / \mathrm{mL}, 75 \mu \mathrm{g} / \mathrm{mL}$, and $100 \mu \mathrm{g} / \mathrm{mL}$ ) of $\mathrm{Nano}_{-\mathrm{SiO}_{2} \text { for }}$ another 24 hours. The equivalent volume of culture medium without $\mathrm{Nano}-\mathrm{SiO}_{2}$ was set as the control group. Five replicate wells were used in each treatment group.

\section{LSCM detection of cellular uptake}

HUVECs were cultured in DMEM in a cell culture dish (35 $\mathrm{mm}$ in diameter) and attached for 24 hours. Then, the HUVECs were treated with red fluorescent-labeled Nano$\mathrm{SiO}_{2}$ for another 24 hours. The cells were washed three times by phosphate buffered saline (PBS) and fixed with $4 \%$ paraformaldehyde for 10 minutes. The cells were then washed several times with $0.1 \%$ Triton $^{\mathrm{TM}} \mathrm{X}-100$ and treated with Actin-Tracker Green (phalloidin-FITC) (Jiancheng, Nanjing, People's Republic of China) for 30 minutes to stain the filamentous actin. Next, 4,6-diamidino-2-phenylindole (DAPI) $(5 \mu \mathrm{g} / \mathrm{mL})$ (Sigma-Aldrich Co., St Louis, MO, USA) was used to stain the nucleus of HUVECs for 5 minutes. After that, the cells in the culture dish were observed under a laser scanning confocal microscope (LSCM) (Leica TCS SP5; Leica Microsystems, Wetzlar, Germany). 


\section{Observation of autophagy by TEM}

The cell samples were fixed overnight in 3\% glutaraldehyde. Then, the samples were washed by $0.1 \mathrm{M} \mathrm{PB}$ for three times and postfixed with $1 \%$ osmic acid for 2 hours. Then, a series of dehydration processes $(50 \%, 70 \%, 80 \%, 90 \%$, and $100 \%$ alcohol, and $100 \%$ acetone) was performed. After that, all the cell samples were embedded in epoxy resin. The thickness of the ultrathin sections was approximately $50 \mathrm{~nm}$, which were made by an ultramicrotome (Ultracut UCT; Leica Microsystems). After being stained by lead citrate and uranyl acetate, these samples were observed under a TEM (JEM-2100; JEOL).

\section{MDC staining}

The fluorescent dye, MDC, is a special marker for autophagic vacuoles. HUVECs were stained with $0.05 \mathrm{mM}$ of MDC (Sigma-Aldrich Co.) for 30 minutes in the dark, after the cells were exposed to Nano-SiO ${ }_{2}$ for 24 hours. Then, the cells were washed with PBS three times. The visualization of MDC staining was detected by LSCM (Leica TCS SP5; Leica Microsystems). Intracellular MDC intensity was quantified by the Volocity Demo 6.1.1 software (PerkinElmer, Inc., Waltham, MA, USA).

\section{Detection of NO/NOS system}

The levels of NO, NOS, endothelial (e)NOS, and inducible (i)NOS) were measured by commercial kits (Jiancheng) according to the manufacturers' protocols. Briefly, after HUVECs were exposed to $\mathrm{Nano}-\mathrm{SiO}_{2}$ with various concentrations $(25 \mu \mathrm{g} / \mathrm{mL}, 50 \mu \mathrm{g} / \mathrm{mL}, 75 \mu \mathrm{g} / \mathrm{mL}$, and 100 $\mu \mathrm{g} / \mathrm{mL}$ ) for 24 hours, the supernatants from the treated groups were collected for detection. Each analysis required that the supernatants were $100 \mu \mathrm{L}$ for measurement. Then, after adding the distilled water and miscellaneous reagents in the text tubes, the $100 \mu \mathrm{L}$ supernatants were mixed and heated in a water bath at $37^{\circ} \mathrm{C}$. The sensitive chromogenic reagents were added into the supernatants at room temperature. After that, the absorbance was measured immediately at $450 \mathrm{~nm}$ (eNOS and iNOS), $550 \mathrm{~nm}$ (NO), and $530 \mathrm{~nm}$ (NOS), using an ultraviolet-visible spectrophotometer (Beckman DU 640B; Beckman Coulter, Inc., Brea, CA, USA).

\section{Proinflammatory cytokine measurement}

The supernatants were collected after the HUVECs were exposed to Nano- $\mathrm{SiO}_{2}$ for 24 hours. Then, the supernatants were centrifuged and stored at $-80^{\circ} \mathrm{C}$ until use. The levels of human $\mathrm{C}$-reactive protein (CRP), human tumor necrosis factor (TNF)- $\alpha$, human interleukin (IL)-1 $\beta$, and human IL-6 were measured by enzyme-linked immunosorbent assay kits (RayBiotech, Inc., Norcross, GA, USA) according to the manufacturer's protocols. Briefly, $100 \mu \mathrm{L}$ of supernatants were added in each well and incubated for 2.5 hours at room temperature. Then, biotin antibody was added to each well and incubated for 1 hour. After that, Streptavidin solution was added and incubated for 45 minutes. Next, the substrate reagent was added and incubated for 30 minutes. After the stop solution was added to each well, the absorbance at 450 $\mathrm{nm}$ was detected immediately using a microplate reader (Thermo Multiskan ${ }^{\mathrm{TM}}$ MK3; Thermo Fisher Scientific).

\section{Western blot analysis}

Equal amounts of $40 \mu \mathrm{g}$ of lysate proteins were loaded onto $12 \%$ sodium dodecyl sulfate-polyacrylamide gels and electrophoretically transferred to polyvinylidene fluoride (PVDF) membranes (EMD Millipore, Billerica, MA, USA). After blocking with nonfat milk (5\%) in Tris-buffered saline (TBS) for 1 hour, the PVDF membrane was incubated, respectively, with LC3, phosphoinositide 3-kinase (PI3K), p-PI3K, protein kinase B (Akt), p-Akt, mammalian target of rapamycin (mTOR), and p-mTOR (CST, USA) (1:1,000, rabbit antibodies) at $4{ }^{\circ} \mathrm{C}$ overnight. Then, the PVDF membrane was rinsed with TBS and Tween 20 (TBST) and incubated with antirabbit immunoglobulin G secondary antibody (CST) for 1 hour. After rinsing with TBST for a total of three times, the proteins bound with the antibody were measured by the enhanced chemiluminescence reagent (Thermo Fisher Scientific). Using the Image $\mathrm{Lab}^{\mathrm{TM}}$ Software (Bio-Rad Laboratories Inc., Hercules, CA, USA), the densitometric analysis of the Western blot results was performed.

\section{Statistical analysis}

The statistical analysis was performed using the SPSS 16.0 software. Data were expressed as the mean \pm standard deviation. Student's $t$-test was performed for comparisons between the two treatment groups. Three or more treatment groups were compared by one-way analysis of variance followed by the least significant difference method for multiple comparisons. All significant differences were considered at the level of $P<0.05$.

\section{Results}

\section{Characterization of Nano-SiO}

The TEM images of Nano- $\mathrm{SiO}_{2}$ exhibited a near-spherical shape with relatively favorable dispersibility (Figure 1). 


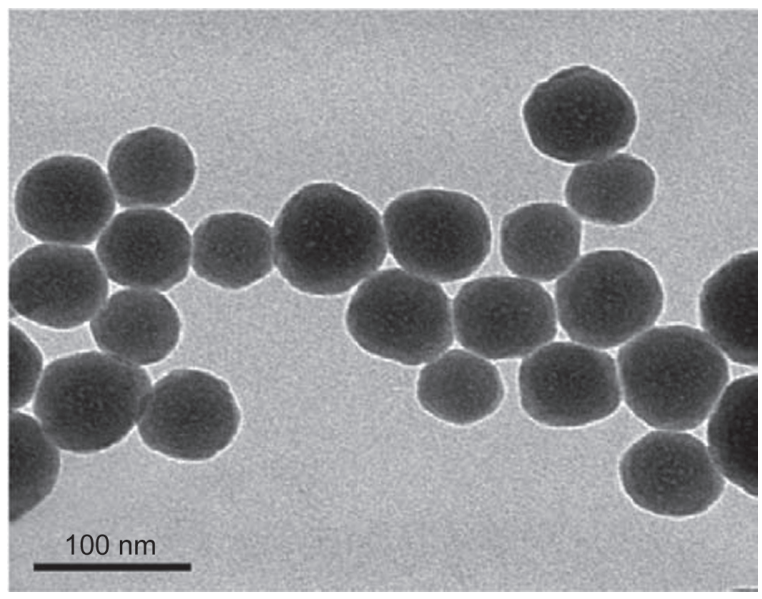

Figure I Characterization of $\mathrm{Nano}-\mathrm{SiO}_{2}$.

Notes: The TEM images of $\mathrm{Nano}_{-} \mathrm{SiO}_{2}$ exhibit a near-spherical shape with relatively favorable dispersibility. The average diameter of $\mathrm{Nano}-\mathrm{SiO}_{2}$ is approximately $62 \mathrm{~nm}$. Abbreviations: $\mathrm{Nano}_{-} \mathrm{SiO}_{2}$, silica nanoparticles; TEM, transmission electron microscopy.

The Nano- $\mathrm{SiO}_{2}$ was fully characterized in our previous studies: ${ }^{14,15}$ the average diameter of the $\mathrm{Nano}^{-\mathrm{SiO}_{2}}$ was approximately $62.1 \pm 7.2 \mathrm{~nm}$. The hydrodynamic sizes and zeta potentials of Nano- $\mathrm{SiO}_{2}$ detected in the different media (distilled water and DMEM) displayed good monodispersity. Finally, the purity of the Nano- $\mathrm{SiO}_{2}$ used in our study was more than $99.9 \%$. Our results confirmed that the Nano- $\mathrm{SiO}_{2}$ possessed favorable dispersibility and stability in the culture medium.

\section{Cellular uptake of $\mathrm{Nano}-\mathrm{SiO}_{2}$}

The cellular uptake and subcellular localization of Nano$\mathrm{SiO}_{2}$ in HUVECs were examined by LSCM. As shown in Figure 2, merged images obtained from LSCM demonstrated that $\mathrm{Nano}-\mathrm{SiO}_{2}$ labeled with red fluorescent dye were taken up by HUVECs after 24 hours of exposure. Furthermore, with the increasing dosage, the numbers of $\mathrm{Nano}-\mathrm{SiO}_{2}$ that were internalized into the HUVECs also increased. Our data showed that Nano-SiO${ }_{2}$ were taken by the endothelial cells in a dose-dependent manner.

\section{Autophagic ultrastructural features induced by $\mathrm{Nano}-\mathrm{SiO}_{2}$}

In accordance with the LSCM results, the cellular uptake and autophagy induction triggered by $\mathrm{Nano}-\mathrm{SiO}_{2}$ were observed in the TEM images by ultrastructural analysis. As shown in Figure 3, HUVECs treated with Nano-SiO 2 exhibited typical autophagic vacuoles with degraded cytoplasmic contents and highly electron-dense $\mathrm{Nano}_{-} \mathrm{SiO}_{2}$ (Figure 3C and D) when compared to the control cells (Figure 3A and B). After careful inspection of the TEM images, the whole process of autophagy induced by Nano- $\mathrm{SiO}_{2}$ was observed in HUVECs: the cytoplasm was engulfed by double-membranous phagophores, which indicated autophagy activation, accompanied by swollen or cristae-rupturing mitochondria (Figure $3 \mathrm{E}$ ); the formation of autophagosomes occurred, which contained cytoplasmic materials inside (Figure 3F); the development of autolysosomes occurred (autophagosome and lysosome fusion) (Figure 3G); and the autolysosomes contained damaged mitochondria and high electron-dense $\mathrm{Nano}-\mathrm{SiO}_{2}$ undergoing degradation at different stages (Figure 3H). Results from the ultrastructural analysis clearly indicated that Nano$\mathrm{SiO}_{2}$ activated the autophagy process in endothelial cells.

\section{MDC staining of autophagic vacuoles}

In order to investigate the activation of autophagy, fluorescent dye MDC was employed to detect autophagic vacuoles in the
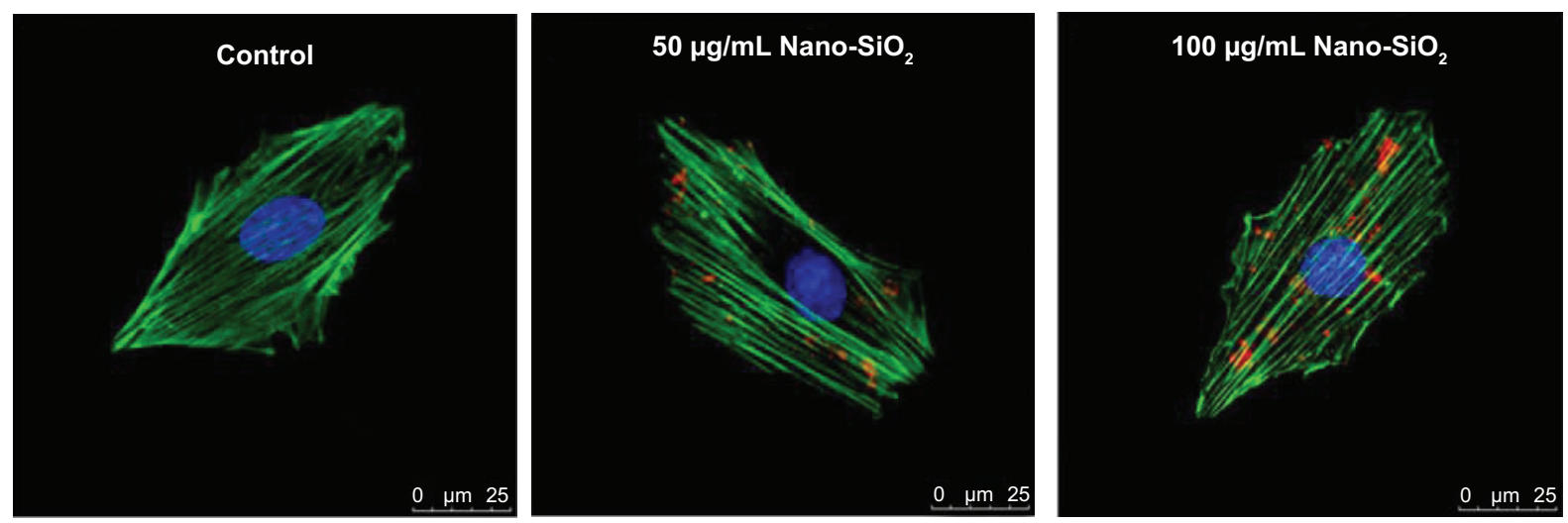

Figure 2 Subcellular localization of $\mathrm{Nano}-\mathrm{SiO}_{2}$.

Notes: LSCM images of HUVECs after incubation for 24 hours with red fluorescent-labeled Nano-SiO ${ }_{2}(50 \mu \mathrm{g} / \mathrm{mL}$ and $100 \mu \mathrm{g} / \mathrm{mL})$. The cell skeleton is stained with Phalloidin-FITC (green), and the cell nucleus with DAPI (blue).

Abbreviations: Nano-SiO , silica nanoparticles; LSCM, laser scanning confocal microscope; HUVEC, human umbilical vein endothelial cells; FITC, fluorescein isothiocyanate; DAPI, 4,6-diamidino-2-phenylindole. 

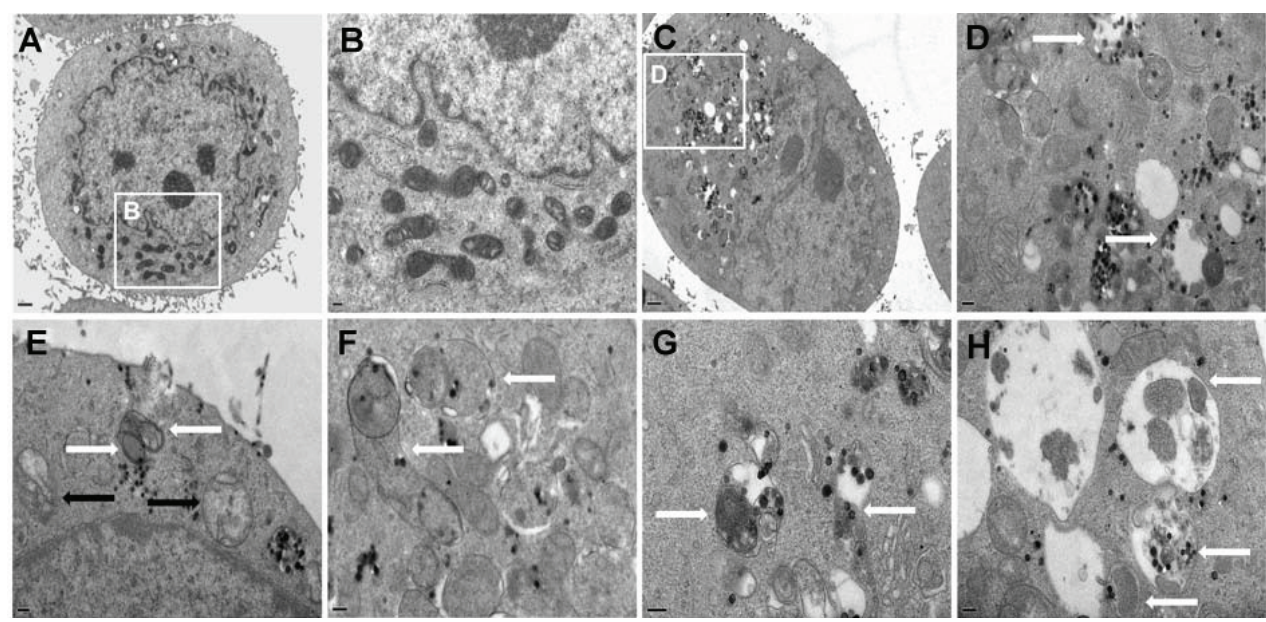

Figure 3 Autophagy activation in HUVECs treated with $\mathrm{Nano}_{-} \mathrm{SiO}_{2}$ measured by ultrastructural analysis.

Notes: (A and B) Untreated cells with normally shaped organelles; (C and D) HUVECs treated with Nano-SiO, showing multiple cytoplasmic vacuoles. Some vacuoles contained electron-dense $\mathrm{Nano}_{-} \mathrm{SiO}_{2}$ and cellular debris (white arrows). Detailed analysis of $\mathrm{HUVECs}$ exposed to $\mathrm{Nano}^{-S i O} \mathrm{O}_{2}$ revealed the clear presence of: (E) doublemembranous phagophores developing into autophagosomes (white arrows) concomitant with swollen or cristae-rupturing mitochondria (black arrows); (F) autophagosomes (white arrows); (G) autolysosomes/amphisomes containing cytoplasmic materials (white arrows); and (H) autolysosomes/amphisomes containing mitochondria and electrondense $\mathrm{Nano}_{-} \mathrm{SiO}_{2}$ undergoing degradation at different stages (white arrows).

Abbreviations: $\mathrm{HUVECs,} \mathrm{human} \mathrm{umbilical} \mathrm{vein} \mathrm{endothelial} \mathrm{cells;} \mathrm{Nano}_{\mathrm{SiO}}$, silica nanoparticles.

HUVECs treated with Nano- $\mathrm{SiO}_{2}$ after 24 hours of exposure. The green fluorescence of the MDC-positive cells was more brightly displayed in the $\mathrm{Nano}-\mathrm{SiO}_{2}$-treated group than in the control group (Figure 4A). Results from the relative fluorescence analysis showed that the fluorescent intensity of MDC-positive cells in the $\mathrm{Nano}-\mathrm{SiO}_{2}$-treated group was significantly elevated (1.76-fold higher than that of the control group) (Figure 4B), which indicated that $\mathrm{Nano}_{-} \mathrm{SiO}_{2}$ triggered autophagy and autophagic vacuole accumulation in the HUVECs.
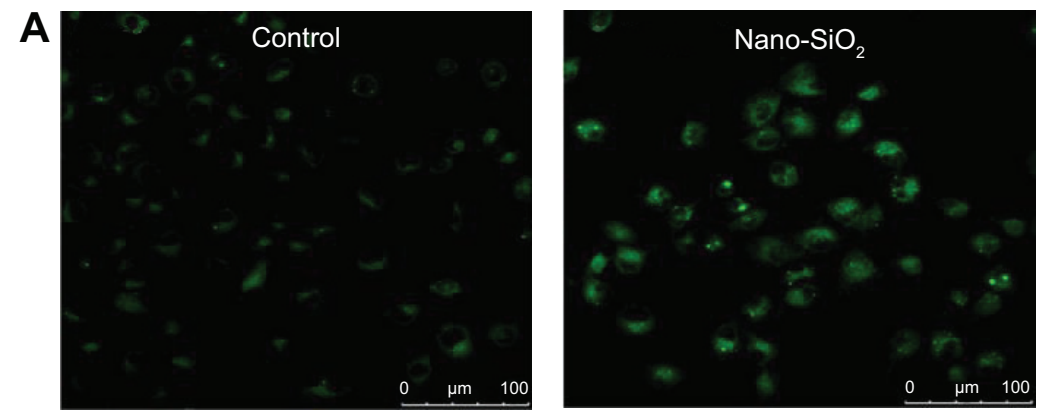

B

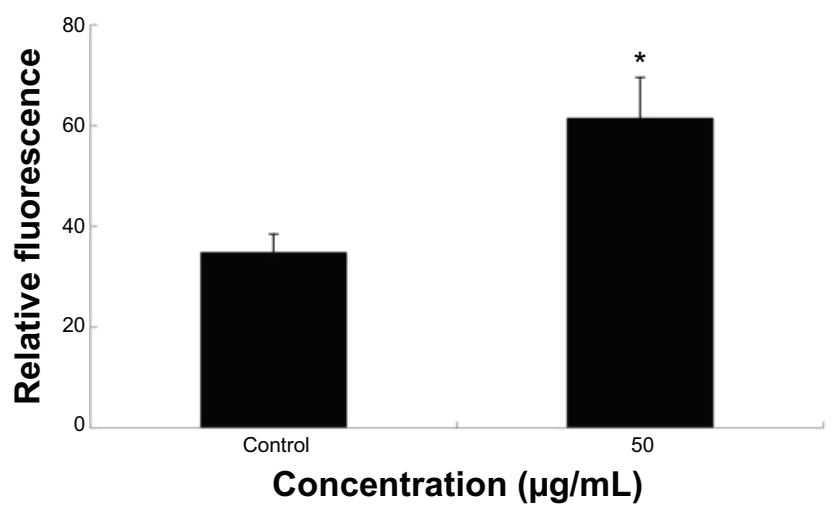

Figure 4 Detection of autophagic vacuoles induced by $\mathrm{Nano}_{-} \mathrm{SiO}_{2}$ via $\mathrm{MDC}$ staining.

Notes: (A) Confocal microscopy images of MDC staining after HUVECs were exposed to $50 \mu \mathrm{g} / \mathrm{mL}$ of Nano-SiO ${ }_{2}$ for 24 hours. (B) Quantification of MDC staining by fluorescent intensity analysis. Student's $t$-test is used for the data analysis. Data are expressed as the mean \pm standard deviation from three independent experiments $(* P<0.05)$. Abbreviations: $\mathrm{Nano}_{-} \mathrm{SiO}_{2}$, silica nanoparticles; MDC, monodansylcadaverine; HUVECs, human umbilical vein endothelial cells. 

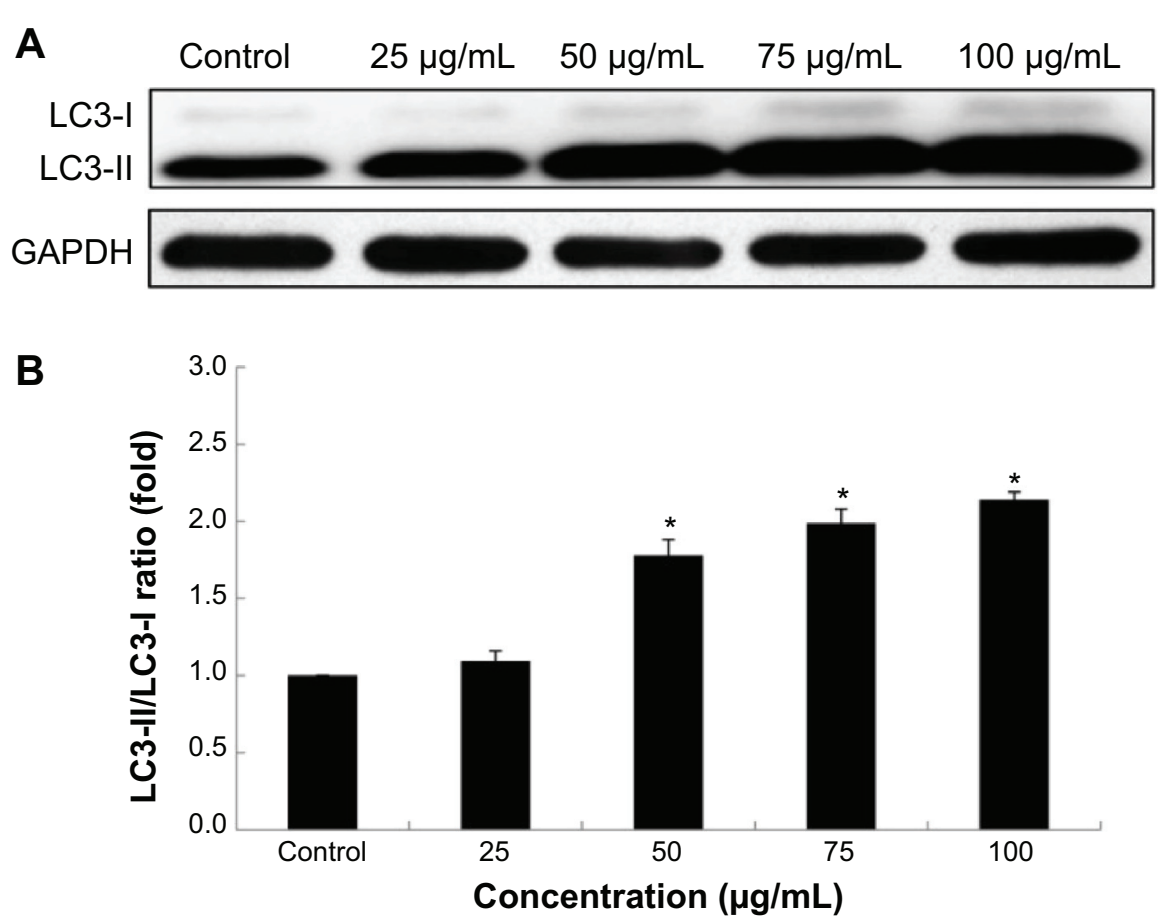

Figure 5 LC3-I/LC3-II conversion in HUVECs treated with Nano-SiO .

Notes: (A) The autophagy marker LC3 protein is detected by Western blot analysis. (B) Relative densitometric analysis showed that the ratio of LC3-II/LC3-I significantly elevated in a dose-dependent manner. ANOVA followed by the LSD method are used for the data analysis. Data are expressed as the mean \pm standard deviation from three independent experiments $(* P<0.05)$.

Abbreviations: LC3, microtubule-associated protein I light chain 3 $\beta$; GAPDH, glyceraldehyde 3-phosphate dehydrogenase; HUVECs, human umbilical vein endothelial cells; Nano-SiO ${ }_{2}$, silica nanoparticles; ANOVA, one-way analysis of variance; LSD, least significant difference.

\section{Detection of autophagy protein biomarker LC3}

Autophagy triggered by Nano- $\mathrm{SiO}_{2}$ was further confirmed by assessing the autophagy protein biomarker, LC3. Western blot analysis demonstrated that the expression of LC3 was enhanced with dosage elevations (Figure 5A). Results from the densitometric analysis showed that the ratio of LC3-II/ LC3-I was increased significantly, which confirmed that the autophagic activity that occurred in HUVECs treated with Nano- $\mathrm{SiO}_{2}$ was enhanced in a dose-dependent manner (Figure 5B).

\section{Effects of $\mathrm{Nano}_{-} \mathrm{SiO}_{2}$ on the NO/NOS system in HUVECs}

To explore the interaction between $\mathrm{Nano}_{-} \mathrm{SiO}_{2}$-induced autophagy and endothelial cell dysfunction, the release of NO and the activities of NOS, eNOS, and iNOS in HUVECs were measured. As shown in Figure 6, when compared to the control group, no significant difference was observed at the lowest concentration $\left(25 \mu \mathrm{g} / \mathrm{mL}\right.$ of $\left.\mathrm{Nano}_{-} \mathrm{SiO}_{2}\right)$ in HUVECs. With increased dosages, the intracellular levels of NO, NOS, and eNOS were significantly decreased; while the level of iNOS was evidently increased in a dose-dependent manner.

\section{Proinflammatory factor induction triggered by Nano-SiO}

To further evaluate the endothelial dysfunction induced by Nano- $\mathrm{SiO}_{2}$, the proinflammatory factors and CRP protein were measured as indicators of an inflammatory response after HUVECs were treated with $\mathrm{Nano}_{-} \mathrm{SiO}_{2}$ for 24 hours. As shown in Figure 7, the secretion of proinflammatory factors (CRP, IL-1 $\beta$, and IL-6) were significantly increased in the $50 \mathrm{mg} / \mathrm{mL}, 75 \mathrm{mg} / \mathrm{mL}$, and $100 \mathrm{mg} / \mathrm{mL}$ Nano-SiO ${ }_{2}$ groups when compared to that of the control. Meanwhile, the release of TNF- $\alpha$ was increased significantly in all Nano-SiO ${ }_{2}^{-}$ treated groups. Our data indicated that $\mathrm{Nano}-\mathrm{SiO}_{2}$ induced the proinflammatory response in a dose-dependent manner.

\section{Effects of Nano-SiO on the PI3K/Akt/ mTOR signaling pathway}

To further understand the mechanisms of $\mathrm{Nano}-\mathrm{SiO}_{2}$ on autophagic activity and endothelial dysfunction, we examined the PI3K/Akt/mTOR signaling pathway by Western blot assay. As shown in Figure 8, the total protein of PI3K, Akt, and mTOR had no significant change after HUVECs were exposed to Nano- $\mathrm{SiO}_{2}$ for 24 hours, while the expression of phosphorylated PI3K, Akt, and mTOR were inhibited 

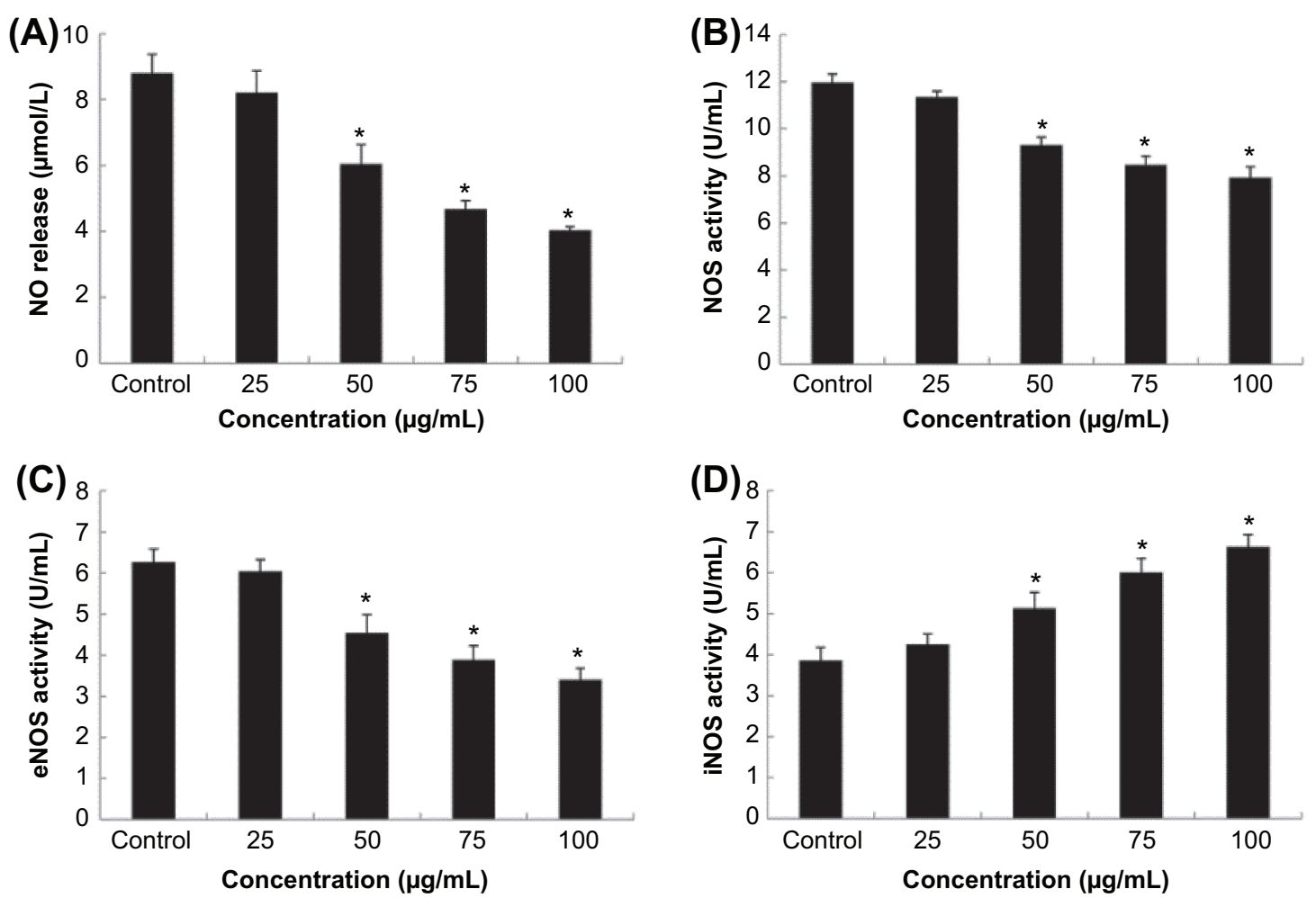

Figure 6 Effects of Nano-SiO, on the NO/NOS system in HUVECs.

Notes: The release of NO (A) and the activity of NOS (B) and eNOS (C) were significantly decreased, while the level of iNOS (D) increased markedly in a dose-dependent manner. ANOVA followed by the LSD method are used for the data analysis. Data are expressed as the mean \pm standard deviation from three independent experiments $(* P<0.05)$.

Abbreviations: NO, nitric oxide; NOS, nitric oxide synthase; eNOS, endothelial nitric oxide synthase; iNOS, inducible nitric oxide synthase; Nano-SiO ${ }_{2}$, silica nanoparticles; HUVECs, human umbilical vein endothelial cells; ANOVA, one-way analysis of variance; LSD, least significant difference.

(A)

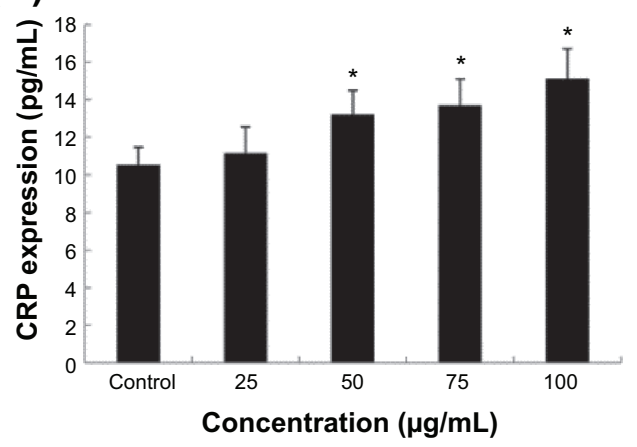

(C)

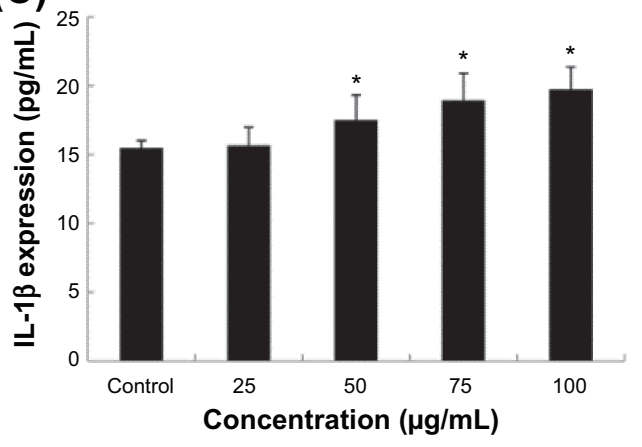

(B)

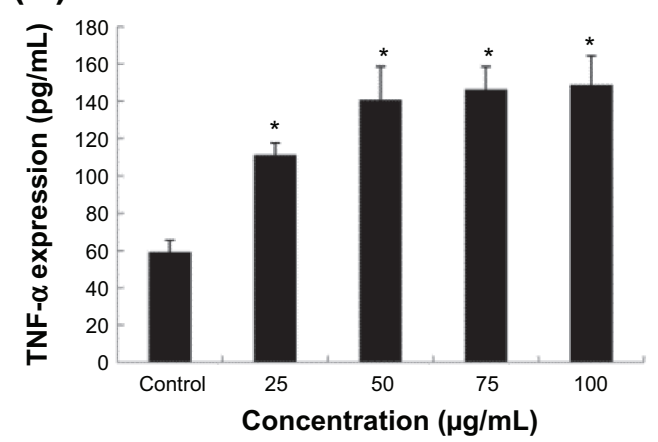

(D)

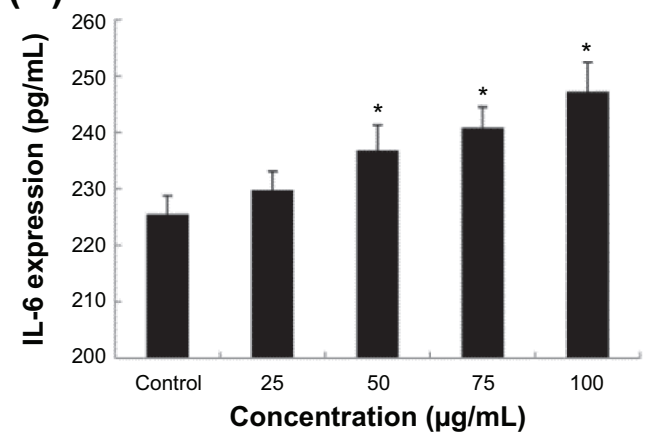

Figure 7 Effects of $\mathrm{Nano}_{-} \mathrm{SiO}_{2}$ on the production of CRP and cytokines in HUVECs.

Notes: The expression of CRP (A) and the production of TNF- $\alpha(\mathbf{B})$, IL-I $\beta$ (C), and IL-6 (D) were significantly increased in a dose-dependent manner. ANOVA followed by the LSD method are used for the data analysis. Data are expressed as the mean \pm standard deviation from three independent experiments $(* P<0.05)$.

Abbreviations: CRP, C-reactive protein; TNF, tumor necrosis factor; IL, interleukin; Nano-SiO, silica nanoparticles; HUVECs, human umbilical vein endothelial cells; ANOVA, one-way analysis of variance; LSD, least significant difference. 

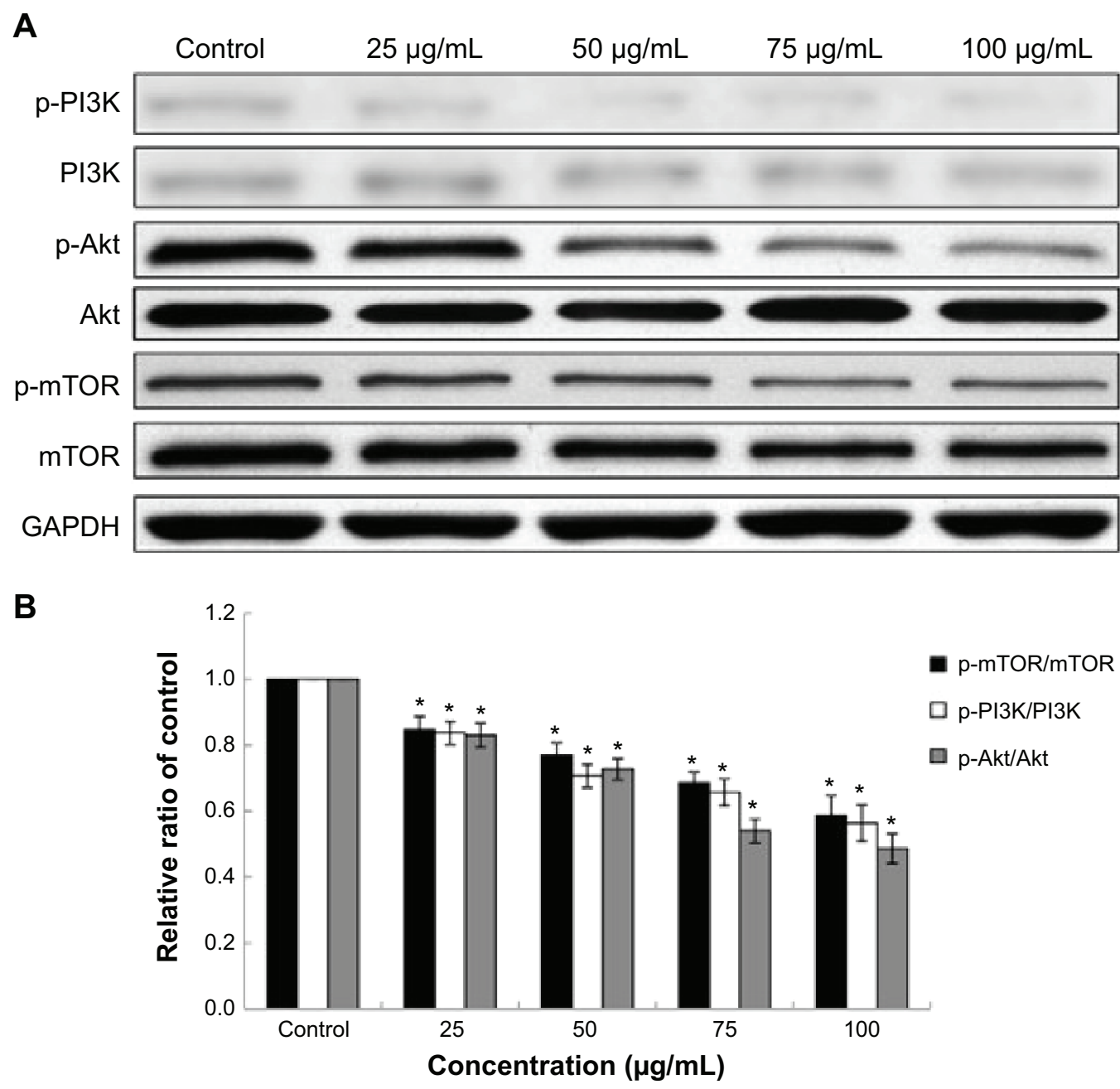

Figure 8 Effects of $\mathrm{Nano}_{-} \mathrm{SiO}_{2}$ on the PI3K/Akt/mTOR signaling pathway.

Notes: (A) The expression of p-PI3K, PI3K, p-Akt, Akt, p-mTOR, and mTOR were measured by Western blot assay. (B) Relative densitometric analysis of the protein bands was performed and presented. Nano-SiO ${ }_{2}$ inhibited the PI3K/Akt/mTOR signaling pathway. ANOVA followed by the LSD method are used for the data analysis. Data are expressed as the mean \pm standard deviation from three independent experiments $(* P<0.05)$

Abbreviations: PI3K, phosphoinositide 3-kinase; Akt, protein kinase B; mTOR, mammalian target of rapamycin; GAPDH, glyceraldehyde 3-phosphate dehydrogenase; Nano-SiO ${ }_{2}$, silica nanoparticles; ANOVA, one-way analysis of variance; LSD, least significant difference.

remarkably in a dose-dependent manner. The downregulation of the PI3K/Akt/mTOR signaling pathway is a typical representation of autophagy activation induced by the Nano$\mathrm{SiO}_{2}$. In addition, PI3K/Akt is also involved in endothelial cell survival, migration, and vascular permeability. The inhibition of $\mathrm{p}$-PI3K and $\mathrm{p}$-Akt expression indicated that Nano- $\mathrm{SiO}_{2}$ could disturb endothelial cell function.

\section{Discussion}

Although the unique physicochemical properties of nanoparticles make them attractive for biomedical applications, the safety evaluation of nanoparticles has always been a challenge. Previously, we had reported that $\mathrm{Nano}-\mathrm{SiO}_{2}$ induced oxidative stress and DNA damage via the Chk1-dependent
G2/M checkpoint signaling pathway in HUVECs. ${ }^{17} \mathrm{We}$ also proved that oxidative stress caused by $\mathrm{Nano}_{-} \mathrm{SiO}_{2}$ led to autophagy and autophagic cell death in HepG2 cells. ${ }^{18}$ However, the relationship between autophagy and endothelial dysfunction remains unclear. In this study, we explored the relationship between $\mathrm{Nano}_{-} \mathrm{SiO}_{2}$-induced autophagic activity and endothelial cell dysfunction, which provided persuasive evidence for the safety evaluation of nano-based products.

The study of the cellular uptake of nanomaterials is necessary for understanding their biological behavior in vitro. ${ }^{19}$ With elevating dosages, a number of $\mathrm{Nano}_{-} \mathrm{SiO}_{2}$ were taken up by HUVECs in a dose-dependent manner (Figure 2). In addition, cellular uptake and the induction of autophagy were observed after HUVECs were treated with $\mathrm{Nano}_{-} \mathrm{SiO}_{2}$ for 
24 hours (Figure 3 ). The typical autophagic ultrastructures and the damaged mitochondria were found in the TEM images (Figure 3), and this was consistent with our previous results that $\mathrm{Nano}-\mathrm{SiO}_{2}$ could induce the depolarization of mitochondria and lead to mitochondrial damage. ${ }^{20}$ It was reported that nanomaterial-induced mitochondrial damage had a close connection with autophagy. ${ }^{21,22}$ The damaged mitochondria can be removed by mitophagy (mitochondriaspecific autophagy). ${ }^{23}$ Given that the vasculature delivers oxygen rather than consumes it, mitophagy plays a key role in vascular biology. To minimize the oxygen consumption in vasculature, one of the potential mechanisms is to reduce the amount of mitochondria through mitophagy. ${ }^{23}$ In this regard, it is not surprising that the number of mitochondria in endothelial cells is less than that in hepatic cells. ${ }^{24,25}$ The entire process of autophagy in endothelial cells exposed to Nano- $\mathrm{SiO}_{2}$ was also present in the TEM images (Figure 3 ), which was similar to the titanium dioxide nanoparticleinduced autophagy noted in keratinocytes, ${ }^{26}$ indicating that different nanomaterials may share a similar pathway to activate autophagy.

In order to further confirm the autophagy activation triggered by Nano- $\mathrm{SiO}_{2}$ in endothelial cells, MDC staining and LC3-I/LC3-II conversion were employed to detect autophagic vacuoles. Results from MDC staining suggested that Nano- $\mathrm{SiO}_{2}$ was the autophagy activator in endothelial cells (Figure 4). The autophagy protein marker, LC3, is converted from cytosolic LC3-I into enzymatic LC3-II when autophagy is activated; thus, the ratio of LC3-II to LC3-I is a standard marker for the detection of autophagy. ${ }^{27,28}$ In this study, we verified that the ratio of LC3-II/LC3-I had increased significantly in a dose-dependent manner (Figure 5). This was also in line with the results of the MDC staining and the ultrastructural analysis in endothelial cells treated with Nano-SiO (Figures 3 and 4). Yet, autophagy acts as a double-edged sword. The abnormal induction of autophagy, either from the upregulated or blocked autophagy flux, will result in toxic effects on endothelial cells and on the vascular system. The ability to maintain normal intracellular homeostasis will be reduced if the decrease in mitochondria is below the threshold value in endothelial cells. As a result, this will lead to endothelial cell dysfunction and the disruption of vascular homeostasis. ${ }^{24}$

Given that the NO/NOS system is directly related to endothelial cell functions, ${ }^{29}$ we measured the level of NO and the activities of NOS, eNOS, and iNOS. NO can be synthesized by the synthase, which is produced in endothelial cells. The NO/NOS system is one of the initial events that occurs in microvascular endothelial cells to maintain homeostasis. ${ }^{30}$ In this study, the results showed that Nano- $\mathrm{SiO}_{2}$ decreased the production of NO and inhibited the activities of NOS and eNOS, while iNOS activity increased (Figure 6). In addition, NO is essential for maintaining endothelial cell functions and vascular homeostasis through the induction of vasorelaxation and the inhibition of platelet adhesion and aggregation. ${ }^{31}$ Disruption of the NO/NOS system leads to vasodilation impairment and inflammatory response, which accelerates the progress of several cardiovascular diseases (eg, atherosclerosis, diabetes, and hypertension). ${ }^{32}$ Meanwhile, recent studies have identified that there was a link between the NO/NOS system and autophagic flux. ${ }^{33}$ Therefore, more studies are needed to investigate the mechanism of the NO/NOS system and the autophagic flux triggered by nanomaterials.

CRP, an independent and important predictor of cardiovascular disease, is stimulated by oxidative stress or proinflammatory cytokines (eg, TNF-a, IL-1 $\beta$, or IL-6). ${ }^{34,35}$ In clinical studies, it was reported that the elevated level of CRP was associated with monocyte infiltration and could promote the progress of atherogenesis by amplifying inflammatory responses. ${ }^{36}$ In the present study, our data demonstrated that Nano- $\mathrm{SiO}_{2}$ could enhance the expression of CRP and the generation of proinflammatory cytokines (TNF-a, IL-1 $\beta$, and IL-6) (Figure 7). Similar to our findings, several studies reported that exposure to nanomaterials could lead to a proinflammatory response in endothelial cells. ${ }^{10,37,38}$ When the primary barrier of the endothelium is disturbed by an inflammatory progress, nanoparticles can impair endothelial and vascular functions, lead to unexpected effects on the human cardiovascular system, and even cause cardiovascular disease. It should be noted that there is crosstalk between autophagy and cytokines in regard to the inflammatory response. ${ }^{39}$ The cytokines (eg, TNF-a, IL-1 $\beta$, and IL-6) have been shown to induce autophagic activity; in contrast, autophagy can regulate the production of proinflammatory cytokines. ${ }^{39} \mathrm{~A}$ bidirectional interaction between autophagy and cytokines can amplify the inflammatory response and lead to endothelial dysfunction.

To gain insight into the mechanism of Nano- $\mathrm{SiO}_{2}-$ induced autopagic activity and endothelial dysfunction, we examined the PI3K/Akt/mTOR signaling pathway by Western blot assay. The serine/threonine kinase mTOR, which is mainly mediated by $\mathrm{PI} 3 \mathrm{~K} / \mathrm{Akt}$ signaling transduction, can negatively regulate autophagy. ${ }^{40} \mathrm{PI} 3 \mathrm{~K}$ and Akt are also involved in vascular endothelial growth factor receptor 2-mediated endothelial cell function. Downregulation of p-PI3K and p-Akt influence endothelial cell survival, 
migration, and vascular permeability. ${ }^{41}$ Results from the Western blot analysis confirmed that the Nano- $\mathrm{SiO}_{2}$ induced endothelial cell dysfunction, as well as activated autophagy (Figure 8). The endothelial cell function is of great importance in maintaining vascular homeostasis. Endothelial dysfunction is an initial event that has been implicated in several cardiovascular diseases. ${ }^{42}$ Thus, more investigations are required to explore the biological mechanisms of the interaction between autophagy, endothelial dysfunction, and cardiovascular diseases triggered by nanomaterials.

\section{Conclusion}

In the present study, the results showed that $\mathrm{Nano}_{-} \mathrm{SiO}_{2}$ can induce autophagic activity, accompanied by NO/NOS system disorder and an inflammatory response, eventually leading to endothelial cell dysfunction via the inhibition of the PI3K/ Akt/mTOR signaling pathway. Our findings confirm that Nano- $\mathrm{SiO}_{2}$-induced endothelial cell dysfunction is associated with autophagy, which may be an important mechanism and adverse outcome pathway of cardiovascular diseases caused by nanoparticles. This will, in turn, be beneficial for the evaluation and reduction of the hazardous effects of nano-based products in medical applications.

\section{Acknowledgments}

This work was supported by the Key Project of National Natural Science Foundation of China (no. 81230065) and Special Project of Beijing Municipal Science and Technology Commission (Z131102002813060, KZ201410025022). The authors thank Professor Wensheng Yang for the preparation of Nano- $\mathrm{SiO}_{2}$.

\section{Disclosure}

The authors report no conflicts of interest in this work.

\section{References}

1. Parveen S, Misra R, Sahoo SK. Nanoparticles: a boon to drug delivery, therapeutics, diagnostics and imaging. Nanomedicine. 2012; 8(2):147-166.

2. Bitar A, Ahmad NM, Fessi H, Elaissari A. Silica-based nanoparticles for biomedical applications. Drug Discov Today. 2012;17(19-20): 1147-1154.

3. Halas NJ. Nanoscience under glass: the versatile chemistry of silica nanostructures. ACS Nano. 2008;2(2):179-183.

4. United States Government Accountability Office. United States Government Accountability Office report on nanotechnology: nanomaterials are widely used in commerce, but EPA faces challenges in regulating risk. Int J Occup Environ Health. 2010;16(4):525-539.

5. Fako VE, Furgeson DY. Zebrafish as a correlative and predictive model for assessing biomaterial nanotoxicity. Adv Drug Deliv Rev. 2009;61(6):478-486.

6. Stern ST, Adiseshaiah PP, Crist RM. Autophagy and lysosomal dysfunction as emerging mechanisms of nanomaterial toxicity. Part Fibre Toxicol. 2012;9:20.
7. Teng RJ, Du J, Welak S, et al. Cross talk between NADPH oxidase and autophagy in pulmonary artery endothelial cells with intrauterine persistent pulmonary hypertension. Am J Physiol Lung Cell Mol Physiol. 2012;302(7):L651-L663.

8. Zabirnyk O, Yezhelyev M, Seleverstov O. Nanoparticles as a novel class of autophagy activators. Autophagy. 2007;3(3):278-281.

9. Suri SS, Fenniri H, Singh B. Nanotechnology-based drug delivery systems. J Occup Med Toxicol. 2007;2:16.

10. Liu X, Sun J. Endothelial cells dysfunction induced by silica nanoparticles through oxidative stress via JNK/P53 and NF-kappaB pathways. Biomaterials. 2010;31(32):8198-8209.

11. Panza JA, Quyyumi AA, Brush JE Jr, Epstein SE. Abnormal endothelium-dependent vascular relaxation in patients with essential hypertension. N Engl J Med. 1990;323(1):22-27.

12. Endemann DH, Schiffrin EL. Endothelial dysfunction. J Am Soc Nephrol. 2004;15(8):1983-1992.

13. Alom-Ruiz SP, Anilkumar N, Shah AM. Reactive oxygen species and endothelial activation. Antioxid Redox Signal. 2008;10(6):1089-1100.

14. Duan J, Yu Y, Shi H, et al. Toxic effects of silica nanoparticles on zebrafish embryos and larvae. PLoS One. 2013;8(9):e74606.

15. Duan J, Yu Y, Li Y, Yu Y, Sun Z. Cardiovascular toxicity evaluation of silica nanoparticles in endothelial cells and zebrafish model. Biomaterials. 2013;34(23):5853-5862.

16. Xu J, Sun L, Li J, Liang J, Zhang H, Yang W. FITC and Ru(phen)32+ co-doped silica particles as visualized ratiometric $\mathrm{pH}$ indicator. Nanoscale Res Lett. 2011;6(1):561.

17. Duan J, Yu Y, Li Y, et al. Toxic effect of silica nanoparticles on endothelial cells through DNA damage response via Chk1-dependent G2/M checkpoint. PLoS One. 2013;8(4):e62087.

18. Yu Y, Duan J, Yu Y, et al. Silica nanoparticles induce autophagy and autophagic cell death in HepG2 cells triggered by reactive oxygen species. J Hazard Mater. 2014;270:176-186.

19. Smith AM, Duan H, Mohs AM, Nie S. Bioconjugated quantum dots for in vivo molecular and cellular imaging. Adv Drug Deliv Rev. 2008;60(11):1226-1240.

20. Sun L, Li Y, Liu X, et al. Cytotoxicity and mitochondrial damage caused by silica nanoparticles. Toxicol In Vitro. 2011;25(8):1619-1629.

21. Afeseh Ngwa H, Kanthasamy A, Gu Y, Fang N, Anantharam V, Kanthasamy AG. Manganese nanoparticle activates mitochondrial dependent apoptotic signaling and autophagy in dopaminergic neuronal cells. Toxicol Appl Pharmacol. 2011;256(3):227-240.

22. Wu YN, Yang LX, Shi XY, et al. The selective growth inhibition of oral cancer by iron core-gold shell nanoparticles through mitochondriamediated autophagy. Biomaterials. 2011;32(20):4565-4573.

23. Green DR, Galluzzi L, Kroemer G. Mitochondria and the autophagyinflammation-cell death axis in organismal aging. Science. 2011; 333(6046):1109-1112.

24. Dromparis P, Michelakis ED. Mitochondria in vascular health and disease. Annu Rev Physiol. 2013;75:95-126.

25. Blouin A, Bolender RP, Weibel ER. Distribution of organelles and membranes between hepatocytes and nonhepatocytes in the rat liver parenchyma. A stereological study. J Cell Biol. 1977;72(2): $441-455$.

26. Zhao Y, Howe JL, Yu Z, et al. Exposure to titanium dioxide nanoparticles induces autophagy in primary human keratinocytes. Small. 2013;9(3): 387-392.

27. Mizushima N, Yoshimori T. How to interpret LC3 immunoblotting. Autophagy. 2007;3(6):542-545.

28. Klionsky DJ, Abdalla FC, Abeliovich H, et al. Guidelines for the use and interpretation of assays for monitoring autophagy. Autophagy. 2012;8(4):445-544.

29. de Fátima A, Zambuzzi WF, Modolo LV, et al. Cytotoxicity of goniothalamin enantiomers in renal cancer cells: involvement of nitric oxide, apoptosis and autophagy. Chem Biol Interact. 2008;176(2-3):143-150.

30. Walshe TE, Connell P, Cryan L, Ferguson G, O’Brien C, Cahill PA. The role of pulsatile flow in controlling microvascular retinal endothelial and pericyte cell apoptosis and proliferation. Cardiovasc Res. 2011;89(3):661-670. 
31. Pacher P, Beckman JS, Liaudet L. Nitric oxide and peroxynitrite in health and disease. Physiol Rev. 2007;87(1):315-424.

32. Corbalan JJ, Medina C, Jacoby A, Malinski T, Radomski MW. Amorphous silica nanoparticles trigger nitric oxide/peroxynitrite imbalance in human endothelial cells: inflammatory and cytotoxic effects. Int J Nanomedicine. 2011;6:2821-2835.

33. Sarkar S, Korolchuk VI, Renna M, et al. Complex inhibitory effects of nitric oxide on autophagy. Mol Cell. 2011;43(1):19-32.

34. Ramage L, Guy K. Expression of C-reactive protein and heat-shock protein-70 in the lung epithelial cell line A549, in response to PM10 exposure. Inhal Toxicol. 2004;16(6-7):447-452.

35. Yuan XM, Li W. Iron involvement in multiple signaling pathways of atherosclerosis: a revisited hypothesis. Curr Med Chem. 2008;15(21): 2157-2172.

36. Fibrinogen Studies Collaboration; Kaptoge S, White IR, Thompson SG, et al. Associations of plasma fibrinogen levels with established cardiovascular disease risk factors, inflammatory markers, and other characteristics: individual participant meta-analysis of 154,211 adults in 31 prospective studies: the fibrinogen studies collaboration. Am J Epidemiol. 2007;166(8):867-879.
37. Schanen BC, Karakoti AS, Seal S, Drake DR 3rd, Warren WL, Self WT. Exposure to titanium dioxide nanomaterials provokes inflammation of an in vitro human immune construct. ACS Nano. 2009;3(9): 2523-2532.

38. Oesterling E, Chopra N, Gavalas V, et al. Alumina nanoparticles induce expression of endothelial cell adhesion molecules. Toxicol Lett. 2008;178(3):160-166.

39. Harris J. Autophagy and cytokines. Cytokine. 2011;56(2):140-144.

40. Pyo JO, Nah J, Jung YK. Molecules and their functions in autophagy. Exp Mol Med. 2012;44(2):73-80.

41. Ferrara N, Gerber HP, LeCouter J. The biology of VEGF and its receptors. Nat Med. 2003;9(6):669-676.

42. Wu CC, Schwartzman ML. The role of 20-HETE in androgen-mediated hypertension. Prostaglandins Other Lipid Mediat. 2011;96(1-4): $45-53$.
International Journal of Nanomedicine

\section{Publish your work in this journal}

The International Journal of Nanomedicine is an international, peerreviewed journal focusing on the application of nanotechnology in diagnostics, therapeutics, and drug delivery systems throughout the biomedical field. This journal is indexed on PubMed Central, MedLine, CAS, SciSearch $®$, Current Contents $\AA /$ Clinical Medicine,

\section{Dovepress}

Journal Citation Reports/Science Edition, EMBase, Scopus and the Elsevier Bibliographic databases. The manuscript management system is completely online and includes a very quick and fair peer-review system, which is all easy to use. Visit http://www.dovepress.com/ testimonials.php to read real quotes from published authors. 